\title{
Ueber tubo-uterine Gravidität nebst Bemerkungen zur Anatomie der Tube ${ }^{1}$.
}

Von

\author{
Dr. Franz Lehmann.
}

(Hierza TafeI XI.)

Unter der Bezeichnung der Graviditas tubo-uterina findet sich in der Literatur eine kleine Anzahl interessanter Fälle, die auch einer verhältnissmässig milden Kritik nur zum geringsten Theile, einer strengen, wie der von Werth ${ }^{2}$ ) überhaupt nicht Stand halten können, sodass nach des Letzteren Ansicht einwandsfreie Fälle, welche die Bezeichnung tubo-uterine Gravidität verdienen, noch nicht berichtet sind.

Wenn wir uns, bevor wir an eine Besprechung der vorhandenen Casuistik gehen, zunächst über die Namengebung einigen wollen, so kann man von einem Sprachgebrauch in diesen Dingen ja wohl nicht reden, aber wir sehen, dass die Autoren mehrfach den Ausdruck ,tubo-uterine Gravidität" für solche Fälle usurpiren, in denen ihrer Annahme nach das Product einer tubaren, im speciellen Falle wohl stets interstitiellen Gravidität per vias naturales, d. h. durch den Uterus und die Scheide, mit mehr oder weniger künstlicher Nachhilfe nach aussen befördert wird.

Schon das Vorkommen solcher Fälle ist zum mindesten sehr selten, sollte man aber den einen oder anderen referirten Fall in dem angeführten Sinne gelten lassen wollen, so scheint mir hierfür der genannte Ausdruck ebensowenig berechtigt, wie man jede geplatzte Tubargravidität, bei der das Ei in die Bauchhöhle gefallen oder ausgestossen ist, als tubo-abdominale Gravidität benennen kann, dazu gehört vielmehr, dass das Ei sich in der Bauchböhle

1) Nach einer Demonstration am 12.3.09 und einem Vortrag am 14.5. 09 in der Gynäk, Geselisch. zu Berlin.

2) Werth, X. Gynäkol. Congress. Würzburg. 1903. 
auch ansiedelt, dort ernährt wird und weiter wächst. Ebenso müsste das primär in der Tube angesiedelte Ei von hier z. T. in den Uterus gelangen, daselbst Wurzel fassen und weiter leben, sodass das Schwangerschaitsproduet theils in der Tube, theils im Uterus sitzt - nicht etwa im Verlaufe der Ausstossung eine kurze Zeit sich in der Lage erhält - oder aber, dass beide Hohlräume zu einem einzigen vereinigt scheinen, in dem wir das Ei finden. Fälle, die mit Sicherheit in diesem Sinne als tubo-uterine Gravidität zu deuten sind, existiren bis jetzt noch nicht, und es ist somit das Präparat, welches ich heute vorlegen möchte, und das so und nur so erklärt werden kann, ein Unicum.

Was die Entstehung der sogenannten tubo-uterinen Gravidität betrifft, so nehmen die älteren Autoren, und auch von den neueren einige, an, dass es sich um ein einfaches Hineinwachsen des im Lumen der Tube angesiedelten Eies durch das Ostium uterinum tubae in den Uterus hinein handle; auf diesem Wege geht, wie z. B. Sehultze auf dem Gynäkologeneongress 1880 sich äussert, wahrscheinlich die tubo-uterine Gravidität in einer Anzahl von Fällen unbemerkt in eine normale Schwangerschaft über, und ähnlich spricht sich Bandl im Lüeke'schen Handbuch aus. In gleicher Weise finde ich von neueren Autoren bei Martin (Krankheiten der Eileiter. I. 77): „Bei der interstitiellen Form sc. der extrauterinen Gravidität kann es vorkommen, dass sich ein Theil des Eies in die Uterushöhle hineinentwickelt, dann handelt es sich um eine Graviditas tubo-uterina.

Auch alle die Autoren, die allein auf klinische Beobachtung sich stützend, Fälle von angeblicher tubo-uteriner Gravidität publicirt haben, und bei denen eine vorher fühlbare Geschwulst vom Charakter eines Adnextumors nach Geburt eines Foetus per vias naturales geschwunden war, müssen wohl von der Vorstellung eines gleichen Vorganges ausgehen, einzelne, wie Eiermann ${ }^{1}$ ), sprechen sich direet in diesem Sinne aus. Mit Recht hält Werth allen diesen entgegen, dass man durch die Palpation allein niemals entscheiden könne, ob es sich um Tubarschwangerschaft, Schwangerschaft im Nebenhorn oder im missbildeten, event. ausgesackten Uterus handelt. Man bedenke nur, wie häufig schon das gravide Corpus uteri für einen tubaren Fruchtsack und die Cervix dabei für den Uterus gehalten worden ist. Vor allen Dingen aber möchte

1) Eiermann, Monatsschr. f. Gebh. u. Gyn. Bd. V. S. 37. 
ich hervorheben, dass in keinem dieser Fälle ein Befund erhoben ist, wie ihn Ruge-Simon ${ }^{1}$ ) als für interstitielle Gravidität - aus der doch jedenfalls die tubo-uterine hervorgehen müsste charakteristisch beschrieben hat. Es sind dies die starke einseitige Graviditätshypertrophie der Musculatur, die Senkrechtstellung. des Fundus, der Hochstand der Adnexe der betroffenen Seite, besonders verglichen mit dem verhältnissmässigen Tiefstande der anderseitigen Adncxe und der Abgang des Ligamentum rotundum lateral von der Geschwulst. Kaum eins von diesen Symptomen ist auch nur andeutungsweise vorhanden. Man müsste also schon annehmen, dass es sich primär gar nicht um interstitielle, sondern um. rein tubare Eiansiedlung gehandelt habe, eine Annahme, die vielleicht gar nicht so paradox ist, wie es im ersten Augenblick erscheint. Aber es fehlt ihnen, wie erwähnt, bei den rein klinischen Befunden überhaupt jede stringento Beweiskraft. Immerhin möchte ich auf einige davon hier kurz eingehen, nämlich um von anderen, noch viel unsichereren zu schweigen, die Fälle von Lwow, Eiermann und Skatsch.

Lwow ${ }^{2}$ ) constatirte bei einer Frau, die ein fieberhaftes Wochenbett durchgemacht hatte, das rechte Ligament gespannt, die rechte Tube verdickt, das linke Ovarium vergrössert. Letzte Menses 10. October 1890. November Gravidität. Uterus gross, teigig, unempfindlich. 5. 2. 1891 Blutungen, wehenartige Schmerzen. Befund: Reehte Unterbauchgegend bis zum Nabel von einer ovalen, prall gespannten, bei Druck empfindlichen Geschwulst eingenommen, welche mit der linken Kante die Mittellinie um $5 \mathrm{~cm}$ nach links überragt. Bei Auftreten der wehenartigen Schmerzen contrahirt sich die Geschwulst, wobei ihre Konturen sichtbar werden. Bei der inneren Untersuchung findet man eine weiche lockere Portio, die nach hinten und links verdrängt ist. Aeusserer Muttermund für einen Finger durchgängig. Uterus vergrössert, gänseeigross, so um seine Achse gedreht, dass die vordere Wand nach links sieht. Rechts dicht am Fundus beginnt die Geschwulst, die die ganze rechte Beckenhälfte einnimmt und nach oben bis zum Nabel reicht.

1) Hugo Simon, Die Graviditas tubo-uterina sowie interstitialis beleuchtet an der Hand des Ruge'schen Symptoms der Fandusdrehung. Diss. inaug. Berlin. 1885.

2) Lwow, Fall von tubo-uteriner Gravidität mit günstigem Ausgang per vias naturales. Medicin. Rundschau, 1891. No. 9, ref. nach Centralbl. 1891. No. 50. S. 1028. 
Bei Zusammenziehungen der Geschwulst fühlt man auch eine Contraction an der rechten Uteruskante. Es liess sich auch das rechte Ligamentum rotundum abtasten und erwies sich die rechte Fundushälfte um das Doppelte grösser als die linke. Die Diagnose lautete daher: Rechtsseitige im 4. bis 5. Monat bestehende tubo-uterine Gravidität. Weiteres Verfahren exspectativ. Tamponade der Scheide gegen Blutungen. 13. 2. Ausstossung des Abortes. Fötus entsprach dem 6. Monat. Nachgeburt in toto ausgestossen. Weiterer Verlauf günstig, während des Wochenbettes allmähliche Verkleinerung der rechten Tube, bei der Entlassung, 27 Tage nach der Geburt, war sie gänsefederkieldick.

Dass aus einer solchen Beobachtung sichere Schlüsse sich nicht ziehen lassen, wird wohl Jeder zugeben müssen, und ich bringe sie nur als Typ einer Reihe von Publicationen. Schwangerschaft eines theilweise fixirten und gedrehten Uterus ist gewiss nicht mit Sicherheit auszuschliessen, und vor Allem die Ausstossung einer 6 monatlichen Frucht durch einen gänseeigrossen Uterus zum mindesten überaus auffallend. Aehnlich steht es mit dem Fall Eiermann, bei dem die Möglichkeit einer Schwangerschaft im rudimentären Horn eines Uterus bicornis absolut nicht von der Hand zu weisen ist; ich referire den Fall, weil er theilweise in der Litteratur anerkannt wird. Eiermann fand bei der Ausräumung eines 4 monalichen Abortes einen auffallend breiten Uterus, der rechts höher stand, als links. Bei der inneren Austastung kommt man zunächst in eine normale Uterushöhle. Rechts oben aber, wo normalerweise die Tube mündet, ist eine kreisrunde, scharfrandige Oeffnung von über Markstückgrösse, die in eine zweite Höhle von wurstförmiger Gestalt, dünnen Wandungen und einer Länge von $7 \mathrm{~cm}$ führt. An ihrer hinteren Fläche sitzt die Placenta. Der Abgang einer Tube von diesem Körper wird nicht wahrgenommen, links dagegen ist die Tube deutlich fühlbar. Die Nebenhöhle sackt sich jenseits des straffen Muskelringes, der den Zugang bietet, allseitig aus; auf der der grossen Höhle zugewandten inneren Seite springt dieser Ring oben, vorn und hinten gegen die Funduswände vor, während er unten direct in die seitliche Corpuswand übergeht. . Nach ausführlicher Besprechung aller in Betracht kommenden Einzelheiten giebt übrigens der Autor selbst zu, dass er den mathematisch strikten Beweis für das Bestehen einer Graviditas tubouterina in seinem Falle schuldig bleiben müsse. 
Skutsehl) schliesslich beobachtete den Abgang eines geschlossenen Decidualsackes und hinterher eines abgestorbenen jungen Eies; fühlbare Verdickung des einen Tubenwinkels an dem entleerten, vor Ausstossung des Eies gleichmässig gestalteten Uterus. Jedenfalls eine Beobachtung, die in keinem Falle beweisend ist, auf die ich aber später noch einmal zurückzukommen habe.

Während diese Fälle schon von Werth im erwähnten Sinne besprochen werden, demonstrirte Wagner²) später, 1905, ein Präparat, welches er ebenfalls als Product einer tubo-uterinen Gravidität deutet: Bei einer Multiparen hatten die Menses 4 Monate sistirt. Links vom Uterus und mit ihm im Zusammenhang findet sich ein leicht beweglicher, nicht druckempfindlicher deutlich fluctuirender Tumor, prall gespannt, von Grösse und Form einer kleinen Wurst. Diagnose: Linksseitiger Tubentumor. 4 Wochen später starke schmerzhafte zweistündige Wehen, danach wird ein wurstförmiger Tumor aus dem Uterus ausgestossen, der nahe an seiner Mitte eine deutliche circuläre Furche aufwies, er enthielt Fruchtwasser und einen 3-4 monatlichen geschrumpften Fötus. Eine nachherige Untersuchung, von der nicht gesagt wird, wann sie vorgenommen wurde, ergab, dass der Adnextumor geschwunden war. Alle gegen die anderen Fälle geäusserten Zweifel treffen auch hier zu, wo schon der Befund eines leichtbeweglichen Tumors neben dem Uterus durchaus gegen interstitielle Schwangerschaft ins Feld geführt werden muss.

Ganz anders stellen sich aber nun die einsehlägigen Verhältnisse dar, wenn wir von den Kenntnissen ausgehen, welche die neueren Untersuchungen über die Eieinbettung in der Tube und speciell deren interstitiellem Theil uns verschafft haben. Das Ei entwickelt sich hier in der auch für die übrige Tube typischen Art. Mangels Ausbildung einer genügend mächtigen Decidua und das gerade im interstitiellen Theil angeblich besonders schwache Epithel wäre hierfür wohl desto minder befähigt — bohrt es sich in die Musculatur der Tube ein und gelangt weiterhin von dem Zeitpunkte an, wo die gewöhnliche Tubengravidität die Tubenmusculatur arrodirend sie durchsetzt und die sog. Ruptur eintritt, seinerseits in die den interstitiellen Theil umgebende Uterusmuscu-

1) Skutsch, VII. Gynäkolog. Congress, Leipzig 1897. Verhandlungen S. 362 .

2) Wagner, Ueber interstitielle Tubargravidität auf Grund von 3 Fällen. Dieses Archiv. Bd. 78. S. 419. 
latur und wird zur eigentlichen intramuralen Schwangerschaft, wie einige diese Zustände auseinanderhalten. Die Tube hat in diesem Stadium kaum noch etwas mit dem $\mathrm{Ei}$ zu thun, sie verläuft, wie angegeben wird, tangential $\mathrm{zu}$ ihm und ist oft garnicht mehr aufzufinden und zu sondiren, weil sie besonders in ihrem uterinwärts gelegenen Antheil seitlich völlig comprimirt wird. Hat sich das $\mathrm{Ei}$ unglücklicherweise an der oberen, hinteren, wenig oder garnicht von Uterusmusculatur bedeckten Wand angesiedelt - der interstitielle Tubentheil verläuft in einem nach hinten oben convexen Bogen -, so kann aber auch jetzt schon die Ruptur stattinden, zu der es sonst erst des Durchwachsens einer dickeren Schicht der an dieser Stelle stets starke Graviditätshypertrophie zeigenden Uterusmusculatur bedürfen würde. Bei Betrachtung der bezüglichen mikroskopischen Präparate werden wir freilich für diese frübzeitige Ruptur auch noch eine andere Ursache kennen lernen.

Richtet sich aber nun weiterhin die Wachsthumstendenz des bereits intramural gelegenen Eies nicht nach aussen, der Serosa zu, sondern nach innen in Richtung auf das Cavum uteri, so erreichen die Zotten schliesslich das letztere, in ihm die Decidua extrauterina, arrodiren sie, wie sonst die Serosa, fressen sich hindurch and können so eine offene Communication zwischen der intramusculär gelegenen, das $\mathrm{Ei}$ enthaltenden Höhle und dem Uteruscavum herstellen. Man sieht leicht ein, dass diese Entwicklung dann eintreten wird, wenn die Placenta, so bald schon von ihrer Entwicklung die Rede sein kann, von der ja die Destruction, das wuchernde Vorwärtswachsen ausgeht. medianwärts - cavumwärts - sich ausgebildet hat. Das übrige Ei steht nur unter dem allgemeinen Wachsthumsdruck, der nur dann an einer anderen Stelle die Muskelkapsel sprengen wird, wenn sie ganz besonders dünn ist. In der That finde ich dementsprechend, dass in allen Fällen, wo ein Einbruch in die Uterushöhle stattgefunden hat oder droht, die Placenta der hier vorangehende Theil ist. Ich möchte auf diesen Umstand darum ein besonderes Gewicht legen, weil ich daraus glaube deduciren zu können, dass es auf diesem Wege nicht zu der Umwandlung einer interstitiellen in eine normale ad terminum gelangende intrauterine Schwangerschaft kommen kann. Denn die Placenta verliert nach dem Durchbruch in das Cayum den grössten Theil ihrer Haftläche, die Zotten ragen frei in die Uterushöhle resp. drängen die Decidua extrauterina vor sich her, aus der sie wohl auch den Fötus nicht genügend ernähren 
können. Er kann sich nicht etwa normal uterin weiter entwickeln, sondern stirbt bereits in seiner extrauterinen Höhle ab. Den ganzen hier geschilderten Entwicklungsgang kann man durch Präparate belegen, die durch Operation oder Section gewonnen sind, wenn der Sack nach aussen in die Bauchhöhle oder nach aussen und innen, in Bauchhöhle und Cavum, geplatzt war und die verschiedenen Theile des Eiinhalts nach den verschiedensten Seiten, wohl auch einmal den Fötus durch das Cavum und die Scheide nach aussen entleerte. Dahin gehören die älteren, in allen einschlägigen $\mathrm{Ar}$ beiten, auch von Werth, citirten Fälle von Braxton Hicks (Oeffnung ins Cavum 2 Zoll gross mit missfarbigen fetzigen Rändern, $5 \frac{1}{2}$ Monate alte Frucht durch die Vagina ausgestossen, Placenta noch im Fruchtsack, Tod an innerer Blutung) und Maschke (Fruchtrumpf auf natürlichem Wege abgegangen, Kopf und Placenta durch einen grossen, die obere Fruchtsackwand einnehmenden Riss ausgetreten, im Blut der Bauchhöhle gefunden, Scheidewand zwischen Uterushöhle und Fruchtsack von einem $3 \mathrm{~cm}$ langen Riss mit zackigen, blutig durchtränkten Rändern durchsetzt). Aehnlich liegt der Fall Poppel. Die Genese solcher Fälle können wir an der Hand ähnlicher Präparate, welche aber vor der violenten Ruptur bewahrt geblieben sind, rückwärts verfolgen. So berichtet Weinbrenner ${ }^{1}$ ) über eine Patientin, die von Thorn operirt wurde (Fall 2); hier zeigte am Präparate an einer pfennigstückgrossen Stelle die blutig verfärbte graue Uterusinnenfläche cin Büschel frei hängender Chorionzotten. Auf dem Durchschnitt zeigt sich, dass die Placenta sich hier zwischen die auseinandergewichene Musculatur einschiebt. Es ist dies das bis jetzt beobachtete äusserste Stadium auf diesem Wege, ohne dass es aber auch hier schon zu einer richtigen tubo-uterinen Gravidität gekommen wäre; ein früheres zeigt das Präparat des ersten Weinbrennerschen Falles, wo nach Ausstossung der Decidua uterina aus dem Uterus noch eine papierdünne Membran sich zwischen $\mathrm{Ei}$ und Uterushöhle befindet.

In keinem dieser genau beobachteten, untersuchten und beschriebenen Fälle, um die es sich hier handelt, findet man etwa das Schwangerschaftsproduct in der Tube, oder an deren uterinem Ende eine Andeutung einer beginnenden Erweiterung, durch welche

1) Weinbrenner, Ueber die interstitielle Schwangerschaft. Zeitschr. f. Gyn. Bd. 51. H. 1. 
das Graviditätsproduct seinen Weg in den Uterus nehmen könnte; es hat ja auch die Tube längst verlassen und sitzt paratubar, und das Eibett ist durch dic auseinandergedrängte Uterusmusculatur gebildet.

Wer an die Möglichkeit eines Durchtrittes des Eies durch das eröffnete uterine Tubenostium glaubt, wird freilich leicht den Mangel von anatomischen Präparaten, die diesen Weg demonstriren, erklären. In diesen Fällen müsste natürlich das Schwangerschaftsprodukt im Lumen der Tube verblieben sein und es käme klinisch einfach zum Abort durch den Uterus oder sogar zur Weiterentwicklung des Eies im Uterus, zur normal zu Ende gehenden Schwangerschaft, die beide keinerlei Indicationen zu einem operativen Eingriff, zur Gewinnung des ersehnten beweisenden Präparates geben. Damit ist aber bis auf Weiteres der ganze Vorgang nur ein hypothetischer und darf nicht zum Bestande unserer sicheren Kenntnisse gerechnet werden, wenn wir auch zugestehen müssen, dass wir im tubaren Abort, den wir so häufig beobachten, ein Analogon für den Abortus per uterum besitzen.

Dies ist bisher der Stand der Dinge gewesen, eine sichere ausgebildete tubo-uterine Gravidität liegt noch nicht vor, und ich gehe nunmehr zu meinem Präparat über, um die weitere Erörterung an dieses anzuschliessen.

Die Patientin, von der das Produkt stammt, war 30 Jahre alt, und schon 2 Jahre vorher wegen Abort und Retroversio in meiner Behandlung. Am 19. 9. 06 erschien sie wieder bei mir mit der Angabe, sie habe am Tage vorher nach 4 monatiger Amenorrhoe abortirt und brachte das Ei mit. Sie wünschte zu wissen, ob alles abgegangen sei, oder sich noch Reste im Uterus befänden. Die genitale Untersuchung ergab: Uterus retrovertirt, rechte Tubenecke vielleicht etwas dick, sonst normal. Aufrichtung leicht. Ring. Noch in Behandlung.

Das Präparat nun, welches in Fig. 1 auf Tafel XI dargestellt jst, stellt, kurz gesagt, einen vollständigen decidualen Ausguss des Uterus dar, dem aussen breitbasig mit einer ca. dreimarkstückgrossen Fläche ein ungefähr wallnussgrosses vollständig erhaltenes Ei, rings von Zotten umgeben, fest und flächenförmig aufsitzt. Weder makroskopisch noch mikroskopiseh sind Muskelfasern zwischen Ei und Decidua zu erkennen. Oberhalb des Eisitzes zeigt der Decidual. sack nach der dem $\mathrm{Ei}$ entsprechenden Seite hin eine rundliche Ausstülpung, in die ich bequem die Nagelphalanx des Ringfingers 
einlegen kann. Reconstruiren wir uns den Hohlraum, in welchem das ganze Gebilde gesessen haben muss, so stellt er eine grössere, an der Eistelle rundlich ausgebuckelte Höble dar.

Was sagt uns nun das Präparat?

Mit Sicherheit können wir jede Schwangerschaft in einer immerwie gearteten und gebildeten Uterushöhle ausschliessen. Im Falle eines missbildeten oder deformirten Uterus, im Falle einer Nebenhornschwangerschaft, stets müsste das Ei vom Decidualsack umschlossen sein, nicht ihm aussen anliegen. Auch einfach umgestülpt ist das Präparat nicht, da ja die rauhe Seite der Decidua nach aussen, nach dem Ei und der Uteruswand hin, die glatte nach innen sieht. Ebensowenig haben wir es aber auch mit einem Augenblicksprodukt zu thun, sondern es handelt sich bei dieser festen und breiten Verwachsung um einen längere Zeit dauernden Zustand, den wir hier vor uns haben, um das Aufgehen des primär extrauterinen Eibettes und der Uterushöhle in einen gemeinsamen Fruchthalterraum, und da das primäre Eibett doch wohl nur die Tube gewesen sein kann, um eine sichere und unbezweifelbare Graviditas tubo-uterina, wenn auch, streng genommen, in dem augenblicklichen Zustande das Fi seinen primären Sitz in der Tube schon verlassen hat.

Bei der Demonstration des Präparates in der Gynäk. Gesellsch. machte mir Hr. Carl Ruge den Einwand, dass es sich möglicherweise um eine intrauterine Gravidität handle, bei welcher nur die Serotina abgerissen und im Uterus zurückgeblieben sei. So bestehe der Sack, dem das Ei ansitzt, aus der Vera + Capsularis (am Ort des Eisitzes) und dieser Capsularis sässe das Ei naturgemäss aussen an. Es ist dieser Einwand in der That als theoretisch möglich anzuerkennen, doch wird er durch das Präparat in keiner Weise gestützt. Zunächst musste das ungefähr wallnussgrosse $\mathrm{Ei}$ die Reflexa soweit vorgetrieben haben, dass man die Aussackung mindestens am frischen Präparat hätte constatiren können; davon war aber aber ebensowenig die Rede, wie jetzt am conservirten Object. Dagegen ist, wie ich hervorhob, eine am Prischen Präparate vorhanden gewesene Ausstülpung nach aussen noch jetzt unverändert zu finden. Sie befindet sich aber oberhalb des Eisitzes und ist auch erheblich dicker, als die den Eiüberzug nach dem Cavum uteri hin bildende Schicht, welche die dünnste Partie des ganzen Sackes darstellt. Auch ist es von rornherein wenig wahrscheinlich, dass die bei dem Alter des Eies doch schon stark 
ausgebildete Serotina, die der am unteren Eirande ca. $5 \mathrm{~mm}$ starken Vera ungefähr an Dicke entsprechen müsste, glatt rings herum genau am Abgang der Capsularis abreissen sollte; mindestens müsste man doch Reste oder die Abrissstelle erkennen. Aber weder makroskopisch noch an den mikroskopischen Präparaten ist davon etwas za erkennen. Auch würde dann an der maternen Eifläche an den Zotten eine mehr oder weniger zusammenhängende Lage von Decidua-Zellen zu erwarten sein, jedoch auch das ist nicht der Fall. Die Capsularis als solche kann man ja an ihrem Bau wohl nicht mit Sicherheit von einer stark durch Druck atrophirten und comprimirten Schicht einer Extrauterina unterscheiden; die Stelle, die ihr nach Ruge's Annahme hier entsprechen würde, ist stark verdünnt und besteht nur aus einer mehrfachen Zellschicht, die theils platt, theils von decidualem Charakter ist und dem Nitabueh'sehen Streifen aufsitzt.

Die grösste Aehnlichkeit mit meinem Präparat hat vielleicht dasjenige, das Skutsch, treilich nicht intakt, sondern in zwei Stücken, Decidualsack und Ei gesondert, so wie sie nacheinander ausgestossen wurden, demonstrirte. Aber gerade darum war er nicht berechtigt, irgendwelche zwingenden Schlüsse zu ziehen. Alle seine Folgerungen trafen nur unter der nicht bewiesenen und für ihn nicht zu beweisenden Voraussetzung zu, dass seine Präparate einst in demselben Zusammenhange standen, wie man ihn hier vor sich sieht.

So sicher wir nun auch hier sagen können, was ist, so sehr sind wir leider über die Art, wie es geworden, aur Vermuthungen angewiesen, die sich nur auf Analogien aufbauen können. Den primär tubaren, und zwar interstitiellen Eisitz können wir per exclusionem noch als sicher festhalten, doch muss es in suspenso gelassen werden, ob sich das Ei im freien Lumen oder einem Blindsack angesiedelt hat. Die Beantwortung dieser Frage ist aber anch nicht von erheblicher Wichtigkeit, da der weitere Verlauf in beiden Fällen derselbe sein musste. Die Lage des Eies wird, wie oben ausführlich auseinandergesetzt, intramural, und nunmehr fressen sich die Zotten, wie schon an den früher erwähnten Specimina zu sehen, bis zur Uterusdecidua durch. Diese ist hier bei Seite gedrängt und stark verdünnt und allmählich ist die ganze musculäre Zwischenwand durch eine so grosse und breite Oeffnung perforirt, dass Eiund Uterushöhle zu einem Ganzen verschmolzen sind. Dies ist der Entwicklungsgang, den ich auf Grund der in anatomischen Präpa- 
raten vorhandenen Vorstadien annehme, und für die die fingerartige Ausstülpung des Sackes oberhalb des Eies, die dem betreffenden Uterushorn entsprechen würde, ebenfalls angeführt werden kann.

Könnte die Entwicklung nun auch anders vor sich gegangen sein, könnte das Ei seinen Weg aus dem freien Lumen der Tube durch deren Ostium uterinum ins Cavum uteri genommen haben? Wie oben mehrfach hervorgehoben, kennen wir kein einziges anatomisches Präparat, welches uns mit Sicherheit ein Ei auf diesem Wege zeigte. Die Annahme dieser Möglichkeit ist rein theoretisch und durch unsere Kenntniss über die Ansiedlung des Eies im interstitiellen Theile durchaus nicht gestützt.

Das Entscheidende bei dieser Frage ist, wie man leicht einsieht, einzig und allein das Verbalten, und zwar besonders die Graviditätsreaction des Tubenepithels an der primären Eiansiedlungsstelle, und ich bemühte mich, etwas Genaueres über die anatomischen Verhältnisse dieser Gegend in der Literatur zu finden. Meine Bemühungen waren aber vergebens, bis mich Herr Robert Meyer darauf aufmerksam machte, dass er in einer Arbeit in der Zeitschrift ${ }^{1}$ ) Einiges darüber angegeben habe. Meyer constatirt zunächst; dass der trichterförmige Uebergang der Tube zum Cavum uteri individuell ausserordentlich verschieden sei, dass sich aber stets die Tube so allmählich erweitert, dass ein derartiges makroskopisches Ostium am Ende der sog. Pars uterina genau zu bezeichnen fast immer unmöglich ist. Das Kriterium für die Grenze sei die mikroskopische Verschiedenheit. Die Sehleimhaut des wirklichen interstitiellen Tubentheils habe keine Drüsen, zeigt dagegen meist den Beginn der Schleimhautfaltung und ausserdem ist der wirkliche interstitielle Tubentheil oftmals gewunden. Ausserdem bewahrt er seine eigene Musculatur, von der die circuläre am weitesten medialwärts sich zu erhalten pflegt. Die Musculatur ist aber keine zuverlässige Erscheinung, da sie individuell zu verschieden ist und sich zuweilen auch als circuläre Schicht noch um die Uterusschleimhaut der Tubenecken legt. - Am maassgebendsten sei die Drüsenlosigkeit der Tuben und danach könne er nach zahlreichen Befunden von der sog. Pars uterina tubae nur etwa das äussere Drittel als wirklichen interstitiellen Tubentheil gelten lassen. Die Drüsen nehmen zwar in den Tubenecken nur allmählich ab,

1) Ueber Drüsen, Cysten, Adenome im Myometrium Erwachsener. Zeitschrift f. Gyn. Bd. 41 . 
sodass die Schleimhaut derselben sich kegelförmig verjüngt, aber die Grenze zum wirklichen interstitiellen drüsenlosen Tubentheil pflegt dennoch eine ziemlich seharfe zu sein. Meyer erkennt also einen intramuralen oder interstitiellen Theil des Sexualcanals an, rechnet aber den grösseren medialen Abschnitt dem Uterus, den kleineren lateralen der Tube $\mathrm{zu}$ und dieser sei nur wenige Millimeter lang.

Um mich von diesen Dingen selbst zu überzeugen, habe ich sie auf einer Reihe von Serienschnitten untersucht, von denen ich einige der wichtigsten hier anführen möchte. Wenn ich auch im Grossen und Ganzen Meyer's Angaben nur bestätigen kann, so möchte ich sie doch noch in dem einen oder anderen Punkte ergänzen. Was zunächst die Musculatur betrifft, so finde auch ich, dass die Ringmusculatur von der Tube bis tief in den Uterus hinein zu verfolgen ist. Es kann also ron einer sphincterenartigen Anordnung am Ostium uterinum tubae nicht die Rede sein. Eine typische uterine Schleimhaut kann man in der That sehr weit lateralwärts finden bis weit in die Gegend, wo die Musculatur nur eine enge runde Röhre von wenigen - hier 3 - Millimetern Durchmesser für die Schleimhaut freilässt (s. Taf. XI, Fig. 3). Diese letztere wiederum hat hier ein ganz kleines schlitzförmiges Lumen, das wir aber doch noch wegen des Schleimhautbaues als zum Uteruscavum gehörig ansprechen müssen. Zugleich aber ist in Fig. 2 ein Präparat abgebildet, an welchem man auch bei mikroskopischer Betrachtung nicht entscheiden kann, ob es sich um Tube oder Uterus handelt, weil die Mucosa hinten schon die Characteristica der Uterina, vorn eher die der Tubaria aufweist. An allerweitesten uterinwärts bleibt an Ober- und Unterwand die Schleimhaut dünn und drüsenlos, weshalb eine Einbettung des Eies an diesen Stellen am schnellsten zu einem Durchfressen der Wandungen und bei der Einbettung oben zur Ruptur führen muss. Jedenfalls finden wir im interstitiellen Theil kein besonders sehwaches Epithel, wie man dies wohl angegeben liest. Was die Länge des interstitiellen Tubentheils betrifft, so finde ich bei meinem einen Fall 7-8 $\mathrm{mm}$ bei directer Messung am Erontalschnitt, bei einem zweiten ea. $6 \mathrm{~mm}$ dureh Berechnung der Serienschnitte.

Was ich abbilde, sind natürlich nur die Verhältnisse eines speciellen Falles und können es nur sein, sicher ist den individuellen Abweichungen der grösste Spielraum gelassen. Aber das Wichtige jst, dass es vorkommt, und wenn auf dieser Grenze zwischen Tube 
und Uterus eine derart dicke, anscheinend doch einer kräftigen decidualen Reaction fähige Schleimhaut existirt, so können wir die Möglichkeit, dass sie das gravide Ei im Lumen der Tube zurückhält, nicht als so ganz unwahrscheinlich bezeichnen und noch immer darauf hoffen, dass ein gütiger Zufall uns einmal das Präparat von einer auf diesem Wege, durch das Ostium uterinum tubae, entstandenen tubo-uterinen Gravidität schenkt; vorläufig jedoch ist nur der andere Weg, der des Durchwachsens einer secundär intramusculären Schwangersehaft, bewiesen, ein Weg, der nunmehr von den ersten Anfängen bis zum erreichten Ziel durch beweisende Präparate belegt werden kann.

Kurz vor Drucklegung der Arbeit finde ich, dass Rémy in No. 104 der Presse médicale 1908 ein dem meinigen analoges Präparat ganz kurz beschrieben hat; ohne weitere Erörterungen setzt er voraus, dass das Ei im Lumen der Tube gesessen habe und von dort direct ausgestossen sei, eine Annahme, über die ich mich oben mit genügender Ausführlichkeit verbreitet habe.

\section{Erklärungen der Abbildungen auf Tafel XI.}

Figur 1. Präparat nach der Natur gezeichnet; man erkennt links oben das von Zotten umgebene Ei, welches dem dicken links unten aufgeschnittenen Decidualsack aufsitzt.

Figur 2. Sagittalschnitt durch die Tuben-Uterusgrenze; nur die hintere Hälfte der Peripherie zeigt Schleimhaut uterinen Charakters; ein kleiner Thei! der vorderen ebenfalls, aber weniger ausgeprägt; der Rest entspricht im Bau der Tube.

Figur 3. Die uterine Schleimhaut ist vollständig ausgebildet; sie liegt in einem Muskelrohr von $3 \mathrm{~mm}$ Durchmesser und ist auch hier aben und unten am schwächsten; die Dicke der Musculatur über ihr beträgt nur ca. $3,5 \mathrm{~mm}$. 
3
$y$
4
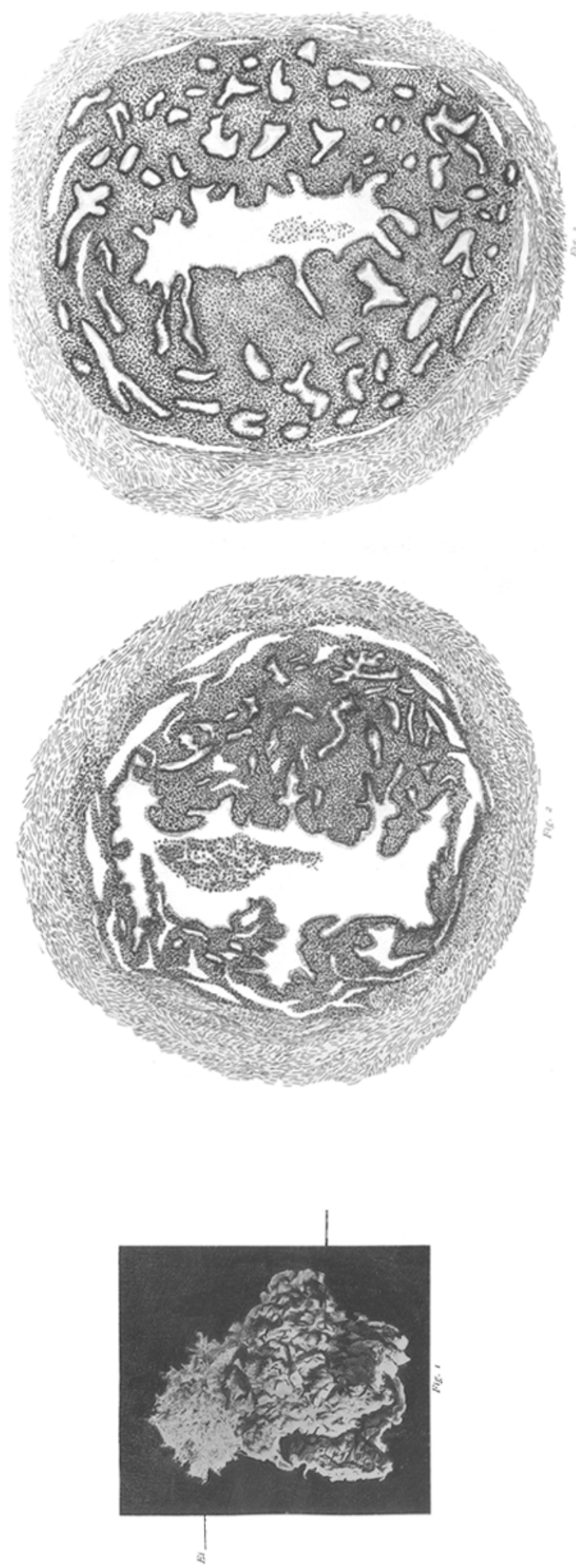